\title{
O conceito de morte e a Síndrome de Asperger ${ }^{1}$
}

\author{
The concept of death and Asperger Syndrome
}

\author{
Letícia Calmon Drummond AMORIM² \\ Francisco Batista ASSUMPÇÃO JUNIOR²
}

\section{Resumo}

O conceito de morte é adquirido paralelamente ao desenvolvimento cognitivo e afetivo da criança, sendo descritos três estágios, paralelos aos estágios piagetianos. O objetivo deste trabalho foi verificar se o conceito de morte em portadores da síndrome de Asperger é similar ao observado em pessoas sem psicopatologia, ou se tem relação com o observado em portadores de deficiência intelectual leve. Para tanto, foram avaliados indivíduos com síndrome de Asperger, indivíduos com deficiência intelectual leve e indivíduos sadios, sem doenças mentais e/ou neurológicas, utilizando-se o Instrumento de Sondagem do Conceito de Morte elaborado por Wilma Torres. Os resultados apontam deficits na aquisição do conceito de morte por indivíduos com síndrome de Asperger, possivelmente relacionados aos deficits na teoria da mente, função executiva e fraca coerência central.

Unitermos: Autismo. Morte. Síndrome de Asperger. Transtorno global do desenvolvimento.

\begin{abstract}
The concept of death is acquired in parallel with cognitive and affective development of children. The objective of this work was it verify if the concept of death in people with Asperger syndrome is similar to that observed in people without psychopathology, or whether it is related to that observed in people with mild mental retardation. For this purpose, individuals with Asperger syndrome, mild mental retardation and healthy individuals without mental and/or neurological diseases were evaluated using the Instrument of Investigation of the Concept of Death developed by Wilma Torres. The results indicated impairment in acquisition of the concept of death in Asperger syndrome cases, possibly related to deficits in the theory of mind, executive function, and weak central coherence.
\end{abstract}

Uniterms: Autism. Death. Asperger's disorder. Pervasive developmental disorder; death.

O autismo é hoje considerado uma síndrome comportamental de base biológica com múltiplas etiologias (Gillberg, 1990). Assim, diversos quadros neurológicos e genéticos são descritos concomitantemente com sua sintomatologia, que é caracterizada por deficits qualitativos na socialização, desenvolvimento da lingua- gem e repertório de interesses e atividades estereotipadas e repetitivas (Organização Mundial de Saúde, 1993).

A Síndrome de Asperger (SA) é caracterizada por prejuízos na interação social e padrões de interesses restritos, não apresentando atraso na aquisição da

$\boldsymbol{\nabla} \mathbf{\nabla} \boldsymbol{\nabla}$

1 Artigo elaborado a partir da dissertação de L.C.D. AMORIM, intitulada "O conceito de morte e a síndrome de Asperger". Universidade de São Paulo, 2008.

2 Universidade de São Paulo, Instituto de Psicologia. Av. Prof. Mello Moraes, 1721, 005508-030, Butantã, SP, Brasil. Correspondência para/Correspondence to: L.C.D. AMORIM. E-mail: <amorimlcd@gmail.com>. 
linguagem nem nas habilidades cognitivas e de autocuidado (Klin, 2006).

Enquanto a prevalência do autismo é descrita ao redor de 6 a 7:1.000 (Fernell \& Gillberg, 2010), a proporção de portadores de SA é de 3:10.000 (Fombonne, 2005) e a relação entre ambos é aventada a partir do conceito de continuum autístico (Wing, 1988), considerando-se o primeiro um comprometimento comportamental cuja constelação sintomatológica é intimamente relacionada ao desenvolvimento cognitivo.

\section{Aspectos cognitivos}

As dificuldades observadas nas relações sociais, na linguagem objetiva e na capacidade de abstração podem ser descritas a partir dos deficits na Teoria da Mente, ou seja, na capacidade de inferir estados mentais a outra pessoa (Baron-Cohen, 1997).

Essa capacidade de perceber o outro e de responder afetivamente à linguagem não verbal auxilia no desenvolvimento da intersubjetividade. Tal aptidão se fundamenta na esfera conativo-afetiva de base biológica, na qual a intencionalidade do bebê em direção ao ambiente se manifesta. Considerando as características do autismo, sugere-se que no bebê autista haveria uma falha nessa condição intencional, levando a dificuldades de simbolização e abstração (Hobson, 1997).

Assim, teorias cognitivas utilizadas para a compreensão do autismo citam a Teoria da Mente, da coerência central e da disfunção executiva. Um deficit cognitivo central levaria à falha no reconhecimento de estados mentais em outra pessoa, habilidade chamada de metarrepresentação ou Teoria da Mente, ocasionando um prejuízo no padrão social, bem como nos padrões simbólico e pragmático. Assim, conceitos e condutas em que se faz necessário atribuir o estado mental ao outro se encontram prejudicados, enquanto aqueles que não requerem metarrepresentações, tais como reconhecimento de sexo, permanência do objeto ou autorreconhecimento no espelho, podem estar intactos. Isso explicaria os padrões alterados de interação social, pragmáticos e simbólicos (Baron-Cohen, 1988).

A dificuldade em juntar partes de informações, formando um todo provido de significados (coesão central), é também característica marcante do autismo característica é um prejuízo no significado global, em favor de um processamento parcial (Hill \& Frith, 2003). A fraca coerência central explicaria a tendência dos portadores da SA em ver partes em lugar de figura inteira, sua resistência a mudanças e também algumas de suas habilidades específicas, como o melhor desempenho nas escalas de Weschler que envolvem reunião e classificação de imagens por série (Happé, 1994; 2001), nas tarefas de localização de figuras escondidas (Shah \& Frith, 1993) e na memorização de uma série de palavras sem sentido, em vez daquelas com significado (Hermelin \& O'Connor, 1970).

A disfunção executiva propõe que os prejuízos na socialização e na comunicação seriam secundários aos deficits nessa função e envolveriam a habilidade para resoluções de problemas e todo o processo que forma a base do comportamento direcionado, como planejamento, memória de trabalho, inibição de respostas e flexibilidade cognitiva (Duncan, 1986). Dessa forma, na SA observam-se deficits nas tarefas que requerem flexibilidade cognitiva e capacidade de planejamento (Hughes \& Russel, 1993; Ozzonoff, Strayer, McMahon \& Filloux, 1994).

Assim, esses aspectos particulares da inteligência se relacionariam diretamente à capacidade de compreensão dos estados mentais próprios e dos outros (Baron-Cohen, 1991).Tal comprometimento prejudicaria não apenas a sociabilidade, mas também a aquisição e percepção de conhecimentos em que se torna necessário transpor o pensamento concreto, entre os quais o conceito de morte.

\section{O conceito de morte}

A compreensão da morte pela criança não é isolada do seu desenvolvimento cognitivo geral. Os três fatores que são relacionados como fundamentais ao estabelecimento do conceito de morte são idade, habilidade cognitiva e vivências de morte (Hunter \& Smith, 2008). Por ser um conceito complexo e abstrato, requer a capacidade de distinguir seres inanimados de animados, significado da constância do objeto (conservação), diferenciação de self e não self e compreensão dos conceitos de tempo e causalidade (Torres, 1978). Assim, é mais plausível considerar que a conceitualização da morte varie de acordo com o nível de desenvolvimento global (Torres, 1996). 
Uma abordagem multidimensional é fundamental para que se tenha uma visão clara do que a criança é capaz de compreender sobre a morte nas diferentes etapas do seu desenvolvimento. Para isso, três dimensões são fundamentais: a irreversibilidade quanto ao caráter definitivo da morte; a universalidade quanto ao fato de que ocorrerá com todos; e a não funcionalidade quanto à compreensão de que todas as funções definidoras da vida cessam com a morte (Speece \&Brent, 1984) .

Kenyon (2001), investigando o conceito de morte nas crianças, refere diversos pontos incongruentes. A universalidade, irreversibilidade, não funcionalidade e mortalidade pessoal em geral seriam conceitos alcançados após os 10 anos de idade em virtude da aquisição do pensamento formal. Assim, o conceito de morte em crianças seria complexo e afetado por vários fatores com os componentes "universalidade" e "irreversibilidade", influenciados pelas habilidades verbais, enquanto "causalidade" e "não funcionalidade" seriam afetadas por experiências pessoais. Questões religiosas, culturais e emocionais também influenciariam o conceito de morte, embora não haja unanimidade quanto a estes fatores, devido à dificuldade em mensurá-los.

Jenkins e Cavanaugh (1985) também relacionoram o desenvolvimento do conceito de morte ao desenvolvimento das habilidades verbais e seu uso no ambiente, enquanto Nunes, Carraro, Jou e Sperb (1998) confirmaram existir relação entre experiências presentes na mídia, nas crenças e nas trocas de vivências e o estabelecimento do conceito de morte.

São descritos três estágios de desenvolvimento do conceito de morte, paralelos aos estágios piagetianos, como indicadores mais fidedignos para avaliação do conceito de morte em crianças (Orbach, Gross, Glaubman \& Berman, 1985; Torres, 1978;). Observando como elas lidam com as dimensões que estabelecem a noção de morte e com a morte propriamente dita, podem-se caracterizar três estágios:

1) estágio pré-operacional: não adquiriram nenhuma dimensão;

2) estágio das operações concretas: compreendem a morte como irreversível e universal;

3) estágio das operações formais: compreendem as três dimensões do conceito de morte.
Os deficits autísticos, ao comprometerem cognitiva e afetivamente a relação com o outro e com o ambiente, têm prejudicado os conhecimentos necessários para ultrapassar o pensamento concreto, comprometendo assim o significado da palavra morte.

Visando compreender melhor o pensamento autístico, principalmente no que se refere à consciência do eu, do outro e do tempo, o objetivo deste trabalho foi estudar como se estrutura o conceito de morte em indivíduos com síndrome de Asperger, comparados com indivíduos com deficiência intelectual leve e saudáveis.

\section{Método}

\section{Participantes}

Foram estabelecidos três grupos pareados para sexo e idade, cada um com 30 sujeitos do sexo masculino (maior incidência de autismo em meninos) e idade superior a 12 anos, quando é esperado que as três dimensões referentes ao conceito de morte tenham sido completamente estabelecidas. Todos os participantes foram instruídos sobre a pesquisa, assinando o Termo de Consentimento Livre e Esclarecido, tendo o projeto sido aprovado pelo Comitê de Ética em Pesquisa do Instituto de Psicologia da Universidade de São Paulo (Parecer $\left.n^{\circ} 2007.005\right)$.

Os grupos foram assim constituídos:

Grupo 1 (Sadios): indivíduos com inteligência na faixa da normalidade e média de 19,5 anos de idade.

Grupo 2 (SA): indivíduos com diagnóstico de SA segundo os critérios do Manual Diagnóstico e Estatístico de Transtornos Mentais - DSM IV TR (American Psychiatric Association, 2002) e escore da Escala de Avaliação Traços Autísticos (ATA) acima de 15, inteligência na faixa de normalidade, avaliada através da Escala de Comportamento Adaptativo de Vineland (Sparrow, Balla \& Cicchetti, 1984), a qual fornece um Quociente de Desenvolvimento (QD), (QD $\geq 70$ Vineland) e idade média de 19,4 anos.

Grupo 3 (DI): indivíduos com diagnóstico de deficiência intelectual leve segundo critérios do DSM IV TR (50 $\leq \mathrm{Q}<70$ Vineland) e média de 19,8 anos de idade. 
Foram considerados critérios de exclusão: comorbidades psiquiátricas, doenças físicas, doenças somáticas crônicas e risco de morte iminente.

\section{Instrumentos}

Os três grupos foram avaliados por meio de:

- Instrumento de Sondagem de Conceito de Morte, elaborado por Torres (1978), com 36 itens que avaliam as dimensões extensão, significado e duração, correspondentes às dimensões universalidade, não funcionalidade e irreversibilidade do conceito de morte biológica. Do total, 11 itens são pertinentes à dimensão extensão, com pontuação máxima de 33 (exemplo: Passarinho morre? Relógio morre?); 17 itens referentes à dimensão significado, com pontuação máxima de 43 (exemplo: Como você sabe se alguém está dormindo ou se está morto? Os mortos comem?); e 8 itens referentes à dimensão duração, com pontuação máxima de 10 (exemplo: Daria comida para pessoa morta? Mandaria enterrar a pessoa morta?).

Os itens pertinentes a cada uma das dimensões pesquisadas foram precedidos por histórias-estímulo, podendo as respostas ser de três tipos: sim/não, sim/não com justificativas e abertas. Os critérios de avaliação compreenderam três categorias: ignorância ou aparente ignorância, noção restrita e noção completa.

- Escala de Avaliação de Traços Autísticos (ATA), utilizada com intuito de diferenciar o grupo SA do grupo DI. A ATA é uma escala construída por Ballabriga, Escudé e Lpaberia (1994) e validada no Brasil (Assumpção Kuczysnki, Gabriel \& Rocca, 1999). Compõe-se de 23 subescalas, cada uma das quais dividida em diferentes itens, que pontuam sintomas de autismo segundo os critérios diagnósticos da Classificação Internacional de Doenças - 10ª edição (CID 10), da Organização Mundial da Saúde (1993) e do DSM-IV-TR (American Psychiatric Association, 2002). Cada subescala recebe pontuação de 0 a 2, conforme a presença ou ausência de sintomas de autismo. Seu escore total é a soma aritmética da pontuação das subescalas, cujo ponto de corte é 15, acima do qual se sugerem sintomas significativos de autismo.

- Escala de Comportamento Adaptativo de Vineland, utilizada para avaliação do nível adaptativo do grupo SA e DI. Essa escala (Speece \& Brent, 1984) é uma entrevista com os pais ou responsáveis, que avalia o comportamento adaptativo. Ao contrário do quociente intelectual, que avalia o melhor desempenho que um indivíduo pode atingir em testes estruturados (Volkmar, 2003), ela avalia o nível funcional da criança em três áreas: comunicação - Valor da Dimen-são Comunicacional da Escala Vineland (VC) (cognição), atividades de vida cotidiana - Valor da Dimensão Atividade da Escala Vineland (VA) (adaptação e independência nas atividades da vida diária e prática) e socialização - Valor da dimensão socialização da escala Vineland (VS), sugerindo um QD. É bastante utilizada tanto para populações com deficiência intelectual como para pessoas com Transtorno Global do Desenvolvimento que, em geral, não respondem aos testes psicométricos (Klin et al., 2007). Sua confiabilidade e validade nessas populações são altas (Bildt, Kraijer, Sytema, \& Minderra, 2005).

Os resultados obtidos entre os três grupos foram comparados por meio de Análise de Variância (Anova) e teste de comparações múltiplas de Bonferroni. O nível de desenvolvimento do grupo SA foi comparado ao do grupo DI, através do teste $t$ independente, com nível de significância 5\% (Maxwell \& Stake, 1997).

\section{Resultados}

A Tabela 1 mostra os pontos em que diferem e em que se assemelham os indivíduos dos grupos SA e DI. Os valores do grupo SA são significativamente superiores em todos os itens avaliados, exceto em VS, relativo à socialização, item em que ambos apresentam o mesmo nível de funcionamento (Tabela 1).

Tabela 1. Comparação entre valores de ATA e Vineland do grupo SA e do grupo DI. São Paulo (SP), 2006-2008.

\begin{tabular}{lcccccc}
\hline & \multicolumn{3}{c}{ AS } & & \multicolumn{3}{c}{$\mathrm{DI}$} \\
\cline { 2 - 3 } \cline { 5 - 6 } & $\mathrm{M}$ & $\mathrm{DP}$ & & $\mathrm{M}$ & $\mathrm{DP}$ \\
\hline ATA $^{*}$ & 34,4 & 3,8 & & 12,7 & 2,2 \\
VT $^{*}$ & 76,9 & 8,1 & & 57,2 & 6,3 \\
VC $^{*}$ & 82,3 & 18,2 & & 31,0 & 9,0 \\
VA $^{*}$ & 76,6 & 14,7 & & 67,8 & 12,0 \\
VS & 87,2 & 15,2 & & 87,5 & 9,1 \\
\hline
\end{tabular}

"p<0,05; M: Média; DP: Desvio-Padrão; ATA: Escala de Avaliação Traços Autísticos; VT: Valor da Escala Vineland Total; VC: Valor da Dimensão Comunicacional da Escala Vineland; VA: Valor da Dimensão Atividade Cotidiana da Escala Vineland; VS: Valor da Dimensão Socialização da Escala Vineland. 
Tabela 2. Escores das três dimensões do conceito de morte nos grupos estudados. São Paulo (SP), 2006-2008.

\begin{tabular}{|c|c|c|c|c|c|c|c|}
\hline & \multicolumn{2}{|c|}{ Sadios $(n=30)$} & \multicolumn{2}{|c|}{ AS $(n=30)$} & \multicolumn{2}{|c|}{$\mathrm{DI}(\mathrm{n}=30)$} & \multirow{2}{*}{$\begin{array}{c}\text { Pontuação } \\
\text { máxima }\end{array}$} \\
\hline & $M$ & DP & $M$ & DP & $M$ & $\mathrm{DP}$ & \\
\hline Extensão & 26,7 & 5,8 & 21,9 & $5,6^{1}$ & 16,7 & $5,5^{1,2}$ & 33 \\
\hline Significado & 39,0 & 3,0 & 24,1 & $8,2^{1}$ & 17,2 & $6,9^{1,2}$ & 43 \\
\hline Duração & 8,6 & 1,2 & 6,6 & $2,0^{1}$ & 5,7 & $2,5^{1}$ & 10 \\
\hline
\end{tabular}

${ }^{1} p<0,05$ em relação ao grupo Sadios; ${ }^{2} p<0,05$ em relação ao grupo SA; M: Média; DP: Desvio-Padrão; SA: Síndrome de Asperger; DI: Diferenças Intelectuais.

NaTabela 2 verificam-se os resultados comparativos entre os grupos do estudo, considerando-se as três dimensões do conceito de morte.

Nas dimensões extensão e significado, o grupo Sadios apresentou maior escore, seguido do grupo SA e, por último, do grupo Dl.

$\mathrm{Na}$ dimensão duração, os valores entre os três grupos obedeceram à hierarquia Sadios, seguido por AS, e DI com a pontuação mais baixa, embora estatisticamente não se tenha evidenciado diferença entre os grupos SA e DI.

\section{Discussão}

O objetivo do presente trabalho foi avaliar o conceito de morte em pessoas com SA, comparando-os a indivíduos sadios e com deficiência intelectual leve. Evidenciou-se que o grupo SA apresentou maior QD que o grupo DI, às custas de um maior rendimento em VC e VA, embora ambos tenham apresentado semelhanças em VS. Esses achados concordam com a definição atual de SA, na qual o desempenho intelectual e as atividades de autocuidado estão preservadas, enquanto a interação social apresenta prejuízos (Klin, 2006).

Os resultados demonstraram que indivíduos com SA apresentam conceito de morte defasado em relação ao grupo composto por indivíduos sadios, mas, quando comparados a indivíduos com deficiência intelectual, apresentam rendimento superior nas dimensões significado e extensão.

Quando comparados tais resultados aos achados de Torres (1978), observa-se que os valores no grupo Dl, em cada dimensão do conceito de morte, aproximaram-se dos valores do grupo de crianças no período pré-operacional. Da mesma maneira, os valores do gru- po SA aproximaram-se dos obtidos para crianças em período de operações concretas, e o grupo Sadios apresentou valores que próximos aos obtidos por crianças com pensamento formal.

Assim, pode-se pensar que os grupos SA e DI apresentam dificuldades na aquisição do conceito de morte. Os dados obtidos concordam com estudo no qual indivíduos com deficiência intelectual apresentaram concepção parcial da noção de morte (McEvoy, MacHale \&Tierney, 2011), por não possuírem estruturas intelectuais nem habilidades fundamentais para o estabelecimento desse conceito.

O significado da palavra morte possui dois componentes: o significado propriamente dito e o sentido, sendo este último influenciado pelas experiências pessoais (Oliveira, 1992). Os estudos de Kenyon (2001), Jenkins \& Cavanaugh (1985) e Nunes et al. (1998) caracterizama ideia de "sentido" como um dos elementos primordiais no desenvolvimento do conceito de morte.

Indivíduos com SA apresentam prejuízo na compreensão da linguagem e falham em comunicar-se funcionalmente, tendendo a ser repetitivos e desconexos, o que acarreta prejuízo na interação social, na troca de experiências e, com base nos resultados do presente estudo, na percepção do conceito de morte.

Tarelho (2005) considerou a fraca coerência central para sugerir que indivíduos com SA são capazes de perceber o estímulo nociceptivo sem o contexto que o acompanha, não considerando dor aquilo que outro indivíduo consideraria. Excluiriam, assim, a dimensão afetiva e social da dor. Essa dificuldade relacionada a conceitos complexos poderia ser explicada por meio da incapacidade de processar informações, devido aos deficits na função executiva e na coerência central, uma vez que a morte seria focada em detalhes, em detrimento da sua visão global. 
Na presente pesquisa, a média do escore do grupo SA para a dimensão significado (não funcionalidade) foi 24,1, similar à das crianças no período de operações concretas do estudo de Torres (1978). Nesse período, ocorre um progresso na conduta e na socialização (Piaget, 1967), iniciando-se a construção lógica que constitui precisamente o sistema de relações que permite a coordenação dos pontos de vista entre si. Assim, o mesmo sistema de coordenações sociais e individuais produz uma moral de cooperação e de autonomia pessoal, em oposição à moral intuitiva de heteronomia, característica das crianças em etapas anteriores de desenvolvimento. $O$ pensamento progride à medida que declina o egocentrismo, aparecendo novas formas de explicação, como a explicação por identificação, com as noções de permanência, tempo, espaço, velocidade e causalidade. Esse pensamento conceitual é o resultado de um jogo de operações coordenadas entre si, em sistemas de conjuntos cuja propriedade mais importante é a de serem reversíveis, em oposição ao pensamento intuitivo da primeira infância.

As crianças em período de operações concretas são capazes de diferenciar seu ponto de vista do dos outros (Piaget, 1968) e também são capazes de perceber o outro como um ser dotado de sentimentos, característica primordial para a socialização. Crianças com SA apresentam deficit na aquisição dessa habilidade, falhando em interagir socialmente e em reconhecer o estado mental das pessoas (Baron-Cohen, 1988). Essa inabilidade inata, caracterizada por problemas de abstração e simbolização, impede-os de atribuir estados mentais ao outro e de prever o comportamento alheio. Dessa forma, embora capazes de perceber a noção de permanência, tempo cronológico, velocidade e espaço, que são padrões pragmáticos e físicos, falham em atingir as noções de temporalidade e morte, já que para tanto é necessária a capacidade de simbolização, abstração e metarrepresentação.

Considerando-se a dimensão duração (irreversibilidade), o escore médio do grupo experimental de SA não apresentou diferenças estatísticas com o dos deficientes intelectuais, o que aponta para um deficit na aquisição dessa dimensão, em que estão implícitas as noções de tempo e temporalidade.

Esses indivíduos, segundo Zukauskas, Nava e 368 Assumpção (2009), identificam termos temporais, noção de período, orientação temporal, porém, no que concerne à temporalidade e à perspectiva que a condição temporal cria na vida de cada um, eles suprimem esse sentido por interações concretas e conceitos espaciais.

Pode-se dizer que o estabelecimento do conceito de morte ocorre paralelamente ao desenvolvimento da estrutura da personalidade da criança. Nos indivíduos com SA, os deficits na interação social prejudicam a motivação que impulsiona o contato com o meio. Dessa forma, tais pessoas são incapazes de experienciar o contato com o mundo de modo amplo (Zukauskas et al., 2009). Seus deficits na teoria da mente fazem com que apresentem uma consciência de identidade estereotipada (Zukauskas et al., 2009) e, assim, não são capazes de compartilhar experiências, o que por consequência altera o significado da palavra morte, principalmente no que tange ao componente sentido.

Assim, a disfunção executiva, a fraca coerência central e os deficits na teoria da mente resultam em inabilidades sociais e dificuldades na interação com as pessoas e com o meio. Diante dessa estrutura deficitária, tais indivíduos apresentam dificuldades no estabelecimento do conceito de morte, o que concorda com os achados de Zukauskas et al. (2009), nos quais a morte foi descrita de forma impessoal, a partir do significado propriamente dito. Em contrapartida, no grupo controle surgiu o sentido da palavra morte e a possibilidade da própria morte.

À medida que as pessoas com SA apresentam dificuldades em vivenciar seu presente e suas escolhas, têm também dificuldade em relacionar passado com futuro a partir dos significados, bem como em planejar projetos que deem razão à sua existência, ocasionando assim um conceito de morte menos elaborado. Isso porque, para se compreender o sentido da palavra morte, são necessárias vivências de perda ou convívio com pessoas que tiveram experiências de perda, e não necessariamente a experiência da morte. Mesmo nos indivíduos com SA que tinham história de perdas na família, embora as médias das três dimensões tenham sido maiores que aquelas do grupo SA, ainda eram inferiores às médias do grupo Sadios deste estudo e do grupo de operações concretas do estudo deTorres (1978).

Os resultados do presente estudo, portanto, indicam que o conceito de morte em indivíduos com SA é prejudicado quando comparado a indivíduos 
saudáveis. Pode-se ainda sugerir que experiências de perda, embora constituam um aprendizado, não suprem os deficits cognitivos dessa população no que se refere ao conceito.

\section{Referências}

American Psychiatric Association. (2002). Manual Diagnóstico e Estatístico de Transtornos Mentais - DSM IVTR (4a ed.). Porto Alegre: Artes Médicas.

Assumpção Jr., F. B., Kuczysnki, E., Gabriel, M. R. \& Rocca, C. C. (1999). Validade e confiabilidade de uma Escala para Detecção de Condutas Autísticas. Arquivos de Neuropsiquiatria, 57 (1), 23-29.

Ballabriga, M. C. J., Escudé R. M. C., \& Lpaberia, E. D. (1994). Escala d'avaluació dels trests autistes (ATA): validez y fiabilidad de uma escala para el examen de lãs conductas autistas. Revista Psiquiatria Infanto-Juvenil, 4, 254-263.

Baron-Cohen, S. (1988) Social and pragmatic deficits in autism: cognitive or affective? Journal of Autism and Developmental Disorders, 18 (3), 379- 401.

Baron-Cohen, S. (1991). The development of a theory of mind in autism: deviance or delay? Psychiatric Clinics of North América, 14 (1), 33-52.

Baron-Cohen, S. (1997). From atencional-goal psychology to bellif-desire psychology: the development of a theory of mind, and its dysfunction. In S. Baron-Cohen, H. TagherFlusberg \& D. J. Coehn. Understanding other minds: perspectives from autism (pp.59-81). New York: Oxford Medical Publication.

Bildt, A., Kraijer, D., Sytema, S., \& Minderra, R. (2005). The psychometric properties of the Vineland adaptative behavior scales in children and adolescents with mental retardation. Journal of Autism and Developmental Disorders, 35 (1), 53-62.

Bosa, C. A. (2001). As relações entre autismo, comportamento social e função executiva. Psicologia: Reflexão e Crítica, 14 (2), 281-287.

Duncan, J. (1986). Disorganization of behavior after frontal lobe damage. Cognitive Neuropsychology, 3 (3), 271-290.

Fernell, E., \& Gillberg, C. (2010). Autism spectrum disorder diagnoses in Stockholm preschoolers. Research in Developmental Disabilities, 31 (3), 680-685.

Fombonne, E. (2005). Epidemiology of autistic disorder and other pervasive developmental disorders. Journal of Clinical Psychiatry, 66 (Suppl 10), 3-8.

Gillberg, G. (1990). Infantile autism: diagnosis and treatment. Acta Psychiatrica Scandinavica, 81 (3), 209-215.

Happé, F. (1994). Autism: an introduction to psychological theory. London: UCL.

Happé, F. (2001). Déficit cognitivo o estilo cognitivo? Coherencia central en autismo. Proceedings of Simposium Internacional Sobre Autismo (pp.105-119). Madrid: Edita Instituto de Migraciones y Servicios Sociales.
Hermelin, B., \&O'Connor, N. (1970). Psychologicalexperiments with autistic children. New York: Pergamon Press.

Hill, E. L., \& Frith, U. (2003). Understanding autism: insights from mind and brain. Philosophical Transactions of The Royal Society of London, Series B, Biological Sciences, 358 (1430), 281-289.

Hobson, P. (1997). Understanding persons: the role of affect. In S. Baron-Cohen, H. Tagher-Flusberg \& D. J. Coehn. Understanding other minds: perspectives from autism (pp.204-227). New York: Oxford Medical Publication.

Hughes, C., Russel, J. (1993). Autistic children's difficulty with disengagement from an object: it's implications for theories of autism. Developmental Psychology, 29 (3), 498-510.

Hunter, S. B., \& Smith, D. E. (2008). Predictors of children's understanding of death: age, cognitive ability, death experience and maternal communicative competence. Omega: Journal of Death and Dying, 57 (2), 143-162.

Jenkins, R. A., \& Cavanaugh, J. C. (1985). Examining the relationship between the development of the concept of death and overall cognitive development. Omega: Journal of Death and Dying, 16 (3), 193-199.

Kenyon, B. L. (2001). Current research in children's conceptions of death: a critical review. Omega: Journal of Death and Dying, 43 (1), 63-91.

Klin, A. (2006). Autismo e síndrome de Asperger: uma visão geral. Revista Brasileira de Psiquiatria, 28 (Supl 1), 3-11.

Klin, A., Saulnier, C. A., Sparrow, S. S., Cicchetti, D. V., Volkmar, F. R., \& Lord, C. (2007). Social and communication habilities and disabilities in higger functioning individuals with autism spectrum disorders: the vineland and the ADDOS. Journal of Autism and Developmental Disorders, 37 (4), 748-759.

Maxwell, D. L., \& Satake, E. (1997). Research and statistical methods in communication disorders (pp.183-189). Philadelphia: Lippincott.

McEvoy, J., MacHale, R., \& Tierney, E. (2011). Concept of death and perceptions of beaverement in adults with intellectual disabilities. Journal of Intellectual Disability Research, 56 (2), 191-203. doi: 10.1111/j.13652788.2011. 01456.x.

Nunes, D. C., Carraro, L., Jou, G. I., \& Sperb, T. M. (1998). As crianças e o conceito de morte. Psicologia: Reflexão e Crítica, 11 (3), 579-590.

Oliveira, M. K. (1992). O problema da afetividade em Vygotsky. In Y. La Taille, M. K. Oliveira \& H. Dantas. Piaget, Vygotsky, Wallon: teorias psicogenéticas em discussão (pp.11-22). São Paulo: Summus.

Orbach, I., Gross, Y., Glaubman, H., \& Berman, D. (1985). Children's perception of death in humans and animals as a function of age, anxiety and cognitive ability. Journal of Child Psychology and Psychiatry, 26 (3), 453-463.

Organização Mundial de Saúde. (1993). Classificação de Transtornos Mentais e de Comportamento da CID 10: descrições clínicas e diretrizes diagnósticas. Porto Alegre: Artes Médicas. 
Ozzonoff, S., Strayer, D. L., McMahon, W. M., \& Filloux, F. (1994). Executive function abilities in autism and tourret syndrome: an information processing approach. Journal of Child Psychology and Psychiatry, 35 (6), 1015-1032.

Piaget, J. (1967). Seis estudos de psicologia. Rio de Janeiro: Forense.

Piaget, J. (1968). A psicologia da criança. São Paulo: Difel.

Shah, A., \& Frith, U. (1993). An islet of ability in autistic children: a research note. Journal of Child Psychology and Psychiatry, 24 (4), 613-620.

Sparrow, S. S., Balla, D. A., \& Cicchetti, D. V. (1984). Vineland adaptive behavior scales: interview edition, expanded form manual. Minnesota: American Guidance Service.

Speece, M. W., \& Brent, B. S. (1984). Children's understanding of death: a review of three components of death concepts. Child Development, 55 (5), 1671-1686.

Tarelho, L. G. (2005). Investigação da percepção dolorosa em pacientes com autismo de alto funcionamento. Dissertação de mestrado não-publicada, Faculdade de Medicina, Universidade de São Paulo.
Torres, W. C. (1978). O conceito de morte em diferentes níveis do desenvolvimento cognitivo: uma abordagem preliminar. Dissertação de mestrado não-publicada, Fundação Getúlio Vargas, Rio de Janeiro.

Torres, W. C. (1996). O desenvolvimento cognitivo e a aquisição do conceito de morteem crianças de diferentes condições sócio-experienciais. Tese de doutorado não-publicada, Universidade Estadual de Campinas.

Volkmar, F. (2003). Ask the editor: adaptative skills. Journal of Autism and Developmental Disorders, 33 (1), 109-110.

Wing, L. (1988). The autistic continuum. In L. Wing. Aspects of autism:biological research (pp.5-8). London: Royal College of Psychiatrists.

Zukauskas, P. R., Nava, S., Assumpção Jr., F. B. (2009). Temporality and Asperger's syndrome. Journal of Phenomenological Psychology, 40 (1),85-106.

Recebido em: 17/3/2010

Versão final reapresentada em: 11/11/2011

Aprovado em: 2/12/2011 